\title{
HETEROGENEITY IN PREFERENCES AND BEHAVIOR IN THRESHOLD MODELS
}

\author{
Philip R Neary \\ Royal Holloway University of London, United Kingdom \\ philip.neary@rhul.ac.uk \\ Jonathan Newton \\ jcsnewton@gmail.com
}

\begin{abstract}
A coordination game is repeatedly played on a graph by players (vertices) who have heterogeneous cardinal preferences and whose strategy choice is governed by the individualistic asynchronous logit dynamic. The idea of potential driven autonomy of sets of players is used to derive results on the possibility of heterogeneous preferences leading to heterogeneous behavior. In particular, a class of graphs is identified such that for large enough graphs in this class, diversity in ordinal preferences will nearly always lead to heterogeneity in behavior, regardless of the cardinal strength of the preferences. These results have implications for network design problems, such as when a social planner wishes to induce homogeneous/heterogeneous behavior in a population.
\end{abstract}

Keywords: Heterogeneity, potential, networks.

JEL Classification Numbers: C72, C73, D02.

\section{INTRODUCTION}

Ever since the classic treatment of Lewis (1969), game theory has concerned itself with the behavior of individuals and groups within societies when interactions between individuals take the form of a coordination game. One area of this literature has studied perturbed adaptive dynamics (Freidlin \& Wentzell,

Both authors declare there are no conflicts of interest. We thank Vincent Crawford, Joel Sobel and Zaifu Yang. Any errors are our own.

Copyright @ () Philip R Neary, Jonathan Newton / 2(1), 2017, 141-159. 
1984; Foster \& Young, 1991) and looked at long run behavior (Young, 1993; Kandori et al., 1993; Blume, 1996; Peski, 2010; Neary, 2012; Staudigl, 2012) and the speed of convergence of behavior (Young, 2011; Ellison, 2000; Montanari \& Saberi, 2010; Newton \& Angus, 2015) in binary-choice coordination games under different interaction structures, which can be represented by graphs, with players represented by vertices and interactions between players represented by edges.

A set of players is said to be autonomous if predictions can be made about the behavior of players within the set without considering the behavior of players outside of the set. Young (2011) shows how one concept of autonomy, potential autonomy, ${ }^{1}$ associated with the maximization of a potential function, is related to graph structure, and uses this connection to derive results on the speed of convergence of a population to homogeneous behavior under loglinear dynamics when interactions are identical, symmetric coordination games. Here, it is shown that when interactions are non-identical and asymmetric, these ideas can be used to make statements about the long run behavior of players, in particular about the possibility of convergence to states in which different players play different strategies. Specifically, there is heterogeneity in players' raw preferences for one action over another, and the strength of any given player's preference is given by an individual-specific preference parameter. Conditions under which heterogeneous preferences lead to heterogeneous behavior are given. In particular, a class of graphs, corpulent graphs, is identified such that, for large enough graphs in this class, random diversity in ordinal preferences will nearly always lead to heterogeneity in behavior, regardless of the cardinal strength of the preferences.

When preferences are homogeneous, long run behavior under log-linear dynamics is independent of interaction structure (Blume, 1996). However, when preferences are heterogeneous, modifying the graph of interactions can affect long run behavior. Consequently, our results have design implications. For example, a planner may wish to design a network of interactions that leads to a particular pattern of behavior, such as the universal adoption of a new technology. Alternatively, a planner may be faced with a given interaction structure but have limited scope to influence the preferences of some of the individuals. We conclude Section 3 with a discussion of such issues.

The paper is organised as follows. Section 2 gives the basic model. Section


from other forms of autonomy. 
3 links heterogeneity and potential autonomy. Section 4 considers random preferences.

\section{MODEL}

The model is a standard one and we follow the notation of Newton \& Sercombe (2017), which builds on that of Young (2011). Consider a simple, finite, connected graph $\Gamma=(V, E)$. The vertex set $V$ represents a set of players. The edge set $E$, consisting of unordered pairs of elements of $V$, represents connections between players. If two vertices share an edge they are said to be neighbors. The number of neighbors of a vertex $i \in V$ is the degree of $i$. For $S \subseteq V, S \neq \emptyset$, denote by $d(S)$ the sum of the degrees of vertices in $S$. For $T, S \subseteq V$, denote by $d(T, S)$ the number of edges $\{i, j\} \in E$ such that $i \in T$ and $j \in S$. For notational convenience we write $d(\{i\})$ as $d(i)$ and $d(\{i\}, S)$ as $d(i, S)$. We write $V \backslash S$ for the complement of $S$ in $V$.

Let $\{A, B\}$ be the (binary) set of strategies available to the players. A strategy profile $\sigma$ is a function $\sigma: V \rightarrow\{A, B\}$ that associates each player with one of the two strategies. Let $\sigma_{S}, \sigma_{-S}$ denote $\sigma$ restricted to the domains $S$ and $V \backslash S$ respectively. Let $\sigma^{A}, \sigma^{B}$ be the strategy profiles such that for all $i \in V, \sigma^{A}(i)=A, \sigma^{B}(i)=B$. Denote by $V_{A}(\sigma) \subseteq V$ the set of players who play strategy $A$ at profile $\sigma$ and by $V_{B}(\sigma) \subseteq V$ the set of players who play strategy $B$ at profile $\sigma$. Given the strategies played by $i$ and $j$, an edge $\{i, j\} \in E$ generates payoffs for $i$ and $j$ as determined by the game in Figure 1. The payoff of player $i \in V$ at profile $\sigma$ is then the sum of these payoffs over the edges he shares with each of his neighbors on the graph. Formally, player $i$ 's payoff at $\sigma$ is

$$
\pi_{i}(\sigma)=\left\{\begin{array}{ll}
\gamma_{i} d\left(i, V_{A}(\sigma)\right) & \text { if } \sigma(i)=A \\
\left(1-\gamma_{i}\right) d\left(i, V_{B}(\sigma)\right) & \text { if } \sigma(i)=B
\end{array} .\right.
$$

This basic setup is identical to the model of Newton \& Sercombe (2017) except for two differences. First, every pairwise interaction is restricted to have zero payoffs off-diagonal. Given that only individual agency is considered in the current paper (i.e. the unit of decision making is always a single player), this is without loss of generality (see the cited work for details). Second, payoffs are allowed to differ between players, whereas the cited work considers symmetric payoff matrices. We refer to $\gamma_{i}$ as player $i$ 's type, which is the (threshold) fraction of $i$ 's neighbours required to play strategy $B$ in order for $i$ 's payoff 


\begin{tabular}{c|c|c|}
\multicolumn{1}{c}{} & \multicolumn{1}{c}{$A$} & \multicolumn{1}{c}{$B$} \\
\cline { 2 - 3 }$A$ & $\gamma_{i}, \gamma_{j}$ & 0 \\
\cline { 2 - 3 }$B$ & 0 & $1-\gamma_{i}, 1-\gamma_{j}$ \\
\cline { 2 - 3 } & &
\end{tabular}

Figure 1: For $\{i, j\} \in E$, for each combination of $A$ and $B$, entries give payoffs for $i \in V$ and $j \in V$ respectively. Both $\gamma_{i}, \gamma_{j} \in(0,1)$.

from playing strategy $B$ to be at least as high as his payoff from playing strategy $A$. Hence the appellation threshold model (Granovetter, 1978).

We note that this specification admits an exact potential function (Monderer \& Shapley, 1996) given by

$$
\text { Potential }(\sigma)=\sum_{\begin{array}{c}
\{i, j\} \in E: \\
\sigma(i)=A \\
\sigma(j)=A
\end{array}}\left(\gamma_{i}+\gamma_{j}\right)+\sum_{\begin{array}{c}
\{i, j\} \in E: \\
\sigma(i)=A
\end{array}} \gamma_{i}+\sum_{\begin{array}{l}
\{i, j\} \in E: \\
\sigma(i)=B
\end{array}} 1
$$

The potential function aggregates information from the game in a way that retains information on the incentives of players under individual agency. Specifically, if we adjust the strategy of any single player, the change in his payoff equals the change in the potential function.

In the current context, the potential function is important in determining long run behaviour under a dynamic process of strategic adjustment. Specifically, we consider the individualistic asynchronous logit dynamic. Let $\sigma^{t}$ denote a strategy profile at time $t \in \mathbb{N}$. At time $t$, a single vertex $i \in V$ is chosen uniformly at random and with probability

$$
\frac{e^{\frac{1}{\eta} \pi_{i}\left(A, \sigma_{-i}^{t-1}\right)}}{e^{\frac{1}{\eta} \pi_{i}\left(A, \sigma_{-i}^{t-1}\right)}+e^{\frac{1}{\eta} \pi_{i}\left(B, \sigma_{-i}^{t-1}\right)}}, \quad \eta>0,
$$

we let $\sigma^{t}(i)=A$. Otherwise we let $\sigma^{t}(i)=B$. For $j \in V, j \neq i$, let $\sigma^{t}(j)=$ $\sigma^{t-1}(j)$.

This process has a unique invariant probability measure $\mu_{\eta}$ on the state space $\{A, B\}^{V}$. Blume (1993) shows that as $\eta \rightarrow 0$, all mass under $\mu_{\eta}$ accumulates on the states $\sigma$ that globally maximize Potential $(\sigma)$. That is, the global maximizers of Potential $(\cdot)$ are the stochastically stable (Young, 1993) states of the process. 


\section{FIXED PREFERENCES AND AUTONOMY}

Adapting the terminology of Newton \& Sercombe (2017), in turn inspired by Young (2011), a set of players $S$ is autonomous if there is some reasonable expectation that players in the set will come to play a subprofile of strategies $\sigma_{S}$ regardless of the behaviour of those outside of $S$. Young (2011) discusses autonomy in terms of potential maximization. Newton \& Sercombe (2017) refer to this form of autonomy as potential autonomy to distinguish it from agency autonomy driven by collective agency. Here we only deal with potential autonomy. A set of players $S$ is $\sigma_{S}^{*}$-autonomous if, for any strategies played by players outside of $S$, a higher potential is attained when players in $S$ play $\sigma_{S}^{*}$ than when they play any other strategies.

Definition 1. $S \subseteq V$ is $\sigma_{S}^{*}$-autonomous if, for all $\sigma$ such that $\sigma_{S} \neq \sigma_{S}^{*}$,

$$
\operatorname{Potential}\left(\sigma_{S}^{*}, \sigma_{-S}\right)>\operatorname{Potential}(\sigma) .
$$

Autonomy will be used to examine the possibility of heterogeneity in preferences leading to heterogeneity in behavior, specifically the possibility of multiple strategies being played at stochastically stable states. Let $S^{A}$ be the set of players who, all else equal, have a preference for strategy $A$, and let $S^{B}$ be the set of players who have a preference for strategy $B$. That is,

$$
S^{A}:=\left\{i \in V: \gamma_{i}>1 / 2\right\} \quad \text { and } \quad S^{B}:=\left\{i \in V: \gamma_{i}<1 / 2\right\}
$$

Let $\sigma^{P}$ be the state at which each player plays his preferred strategy. That is,

$$
\sigma^{P}(i)=\left\{\begin{array}{ll}
A & \text { if } i \in S^{A} \\
B & \text { if } i \in S^{B}
\end{array} .\right.
$$

We now state our first result.

Lemma 1. Fix a graph $\Gamma=(V, E)$ and a set of types $\left\{\gamma_{i}\right\}_{i \in V}$.

[1a] $\sigma^{P}$ is the unique stochastically stable state if and only if $S^{A}$ is $\sigma_{S^{A}}^{A}$ autonomous and $S^{B}$ is $\sigma_{S^{B}}^{B}$-autonomous.

[1b] $\sigma^{A}$ is a stochastically stable state if and only if there does not exist $S \subseteq V$ such that $S$ is $\sigma_{S}^{B}$-autonomous. 


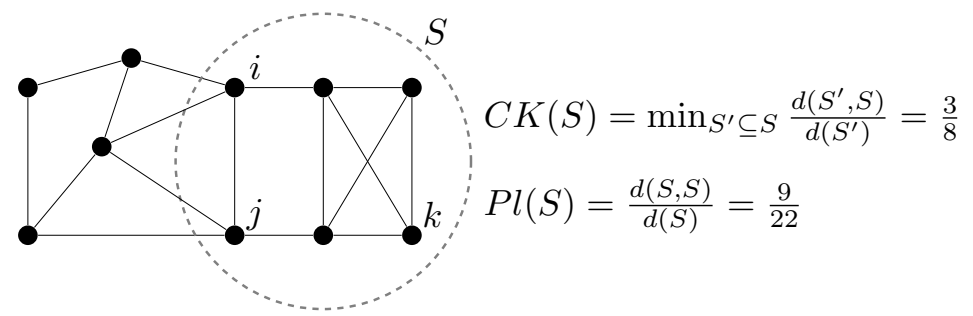

Figure 2: A graph $\Gamma=(V, E)$ and a subset of vertices $S \subset V$ that illustrate aspects of Lemmas 1, 2, 3. See text for discussion.

[1c] $\sigma^{B}$ is a stochastically stable state if and only if there does not exist $S \subseteq V$ such that $S$ is $\sigma_{S}^{A}$-autonomous.

The "if" part of [1a] and the "only if" parts of [1b],[1c] follow immediately from the definition of $\sigma_{S}$-autonomy. The "only if" part of [1a] follows from complementarity of the arguments in the potential function, specifically the fact that if $S^{A}$ is not $\sigma_{S^{A}}^{A}$-autonomous, then $\sigma_{S^{A}}=\sigma_{S^{A}}^{A}$ cannot uniquely maximize potential given $\sigma_{S^{B}}=\sigma_{S^{B}}^{B}$. The "if" parts of [1b],[1c] are proved by showing that if, from $\sigma^{A}$ or $\sigma^{B}$, potential can be increased by changing the strategies of a set of players, then some subset of this set must be autonomous for their new strategies.

If the conditions of neither [1b] nor [1c] are met, that is there exist $S, S^{\prime} \subseteq V$ such that $S$ is $\sigma_{S}^{B}$-autonomous and $S^{\prime}$ is $\sigma_{S^{\prime}}^{A}$-autonomous, then any stochastically stable state will be heterogeneous. However, such a state will only involve each player playing his preferred strategy if the condition in [1a] holds. If neither [1a] nor [1b] nor [1c] holds, then any stochastically stable state will be both heterogeneous and involve some players playing their less preferred strategy.

Considering Figure 2 and ignoring terms that shall be defined later in the paper, we can, for example, state by [1b] that if the subset of vertices $S$ is $\sigma_{S}^{B}$-autonomous, then $\sigma^{A}$ is not a stochastically stable state. Conversely if there exists no such $\sigma_{S}^{B}$-autonomous set (over all subsets of vertices), then $\sigma^{A}$ is stochastically stable.

Young (2011) shows that potential autonomy depends on the graph theoretic property of close-knittedness, which measures how well integrated each subset of a group of players is with the rest of the group. Our precise 
definition of close-knittedness follows Newton \& Sercombe (2017). ${ }^{2}$ The close-knittedness of a set $S \subseteq V$ is given by

$$
C K(S):=\min _{S^{\prime} \subseteq S} \frac{d\left(S^{\prime}, S\right)}{d\left(S^{\prime}\right)} .
$$

Young (2011) links potential autonomy and close-knittedness to discuss the speed of convergence to homogeneous strategy profiles. Under heterogeneous preferences, these connections can be used to make statements about the stochastic stability of heterogeneous strategy profiles. Similarly to Proposition 2 of Young (2011), we obtain the following lemma.

Lemma 2. Fix a graph $\Gamma=(V, E)$ and a set of types $\left\{\gamma_{i}\right\}_{i \in V}$. Then, for any nonempty $S \subseteq V$,

[2a] If $C K(S)>\max _{i \in S} 1-\gamma_{i}$, then $S$ is $\sigma_{S}^{A}$-autonomous.

[2b] If $S$ is $\sigma_{S}^{A}$-autonomous, then $C K(S)>\min _{i \in S} 1-\gamma_{i}$.

[2c] If $C K(S)>\max _{i \in S} \gamma_{i}$, then $S$ is $\sigma_{S}^{B}$-autonomous.

[2d] If $S$ is $\sigma_{S}^{B}$-autonomous, then $C K(S)>\min _{i \in S} \gamma_{i}$.

That is, sets $S$ with high $C K(S)$ are more likely to be potential autonomous and vice versa. The min and max operators enter because of the heterogeneity of the values of $\gamma_{i}$ for $i \in S$. High values of $\gamma_{i}$ make $i \in S$ more amenable to playing $A$, and low values do the opposite. As, by definition, $C K(S) \in[0,1 / 2]$ and $\gamma_{i} \in(0,1)$, it can never be the case that both $C K(S)>1-\gamma_{i}$ and $C K(S)>\gamma_{i}$, so the conditions in [2a] and [2c] never hold simultaneously.

Returning to Figure 2, we see that $C K(S)=3 / 8$. Consequently, by [2c], if every $l \in S$ has type $\gamma_{l}<3 / 8$, then $S$ is $\sigma_{S}^{B}$-autonomous. Conversely, [2d] tells us that if $S$ is $\sigma_{S}^{B}$-autonomous, then at least one player $l \in S$ has $\gamma_{l}<3 / 8$. The reason that the converse does not imply the inequality for all players in $S$ is that the subset $S^{\prime} \subseteq S$ that determines the value of $C K(S)$ need not include all of the players in $S$. In Figure 2, we see that the minimum over $d\left(S^{\prime}, S\right) / d\left(S^{\prime}\right)$ is attained when $S^{\prime}=\{i, j\}$. The constraints on type that $\sigma_{S}^{B}$-autonomy of $S$ places on vertices such as $k$ which lie outside of this subset are less tight.

If we restrict the set of types, $\left\{\gamma_{i}\right\}_{i \in V}$, so that there are two types of player, those with $\gamma_{i}=\gamma_{A}>1 / 2$ who prefer strategy $A$, and those with $\gamma_{i}=\gamma_{B}<1 / 2$

2 Young (2011) refers to a set $S$ as ' $r$-close knit' if $C K(S) \geq r$. 


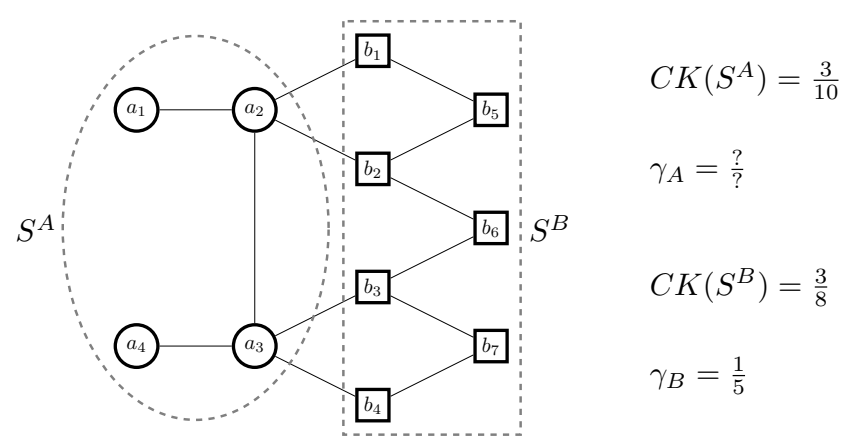

Figure 3: A graph $\Gamma=(V, E)$ and a subset of vertices $S \subset V$. As per the assumptions of Corollary 1 , there are two types, $\gamma_{A}$ and $\gamma_{B}$, with $\gamma_{B}<1 / 2<\gamma_{A}$. Consequently, for $i \in S^{A}=\left\{a_{1}, \ldots, a_{4}\right\}, \gamma_{i}=\gamma_{A}$ and for $i \in S^{B}=\left\{b_{1}, \ldots, b_{7}\right\}$, $\gamma_{i}=\gamma_{B}$. In the text we use this example to illustrate the use of Lemmas 1,2 and Corollary 1 in the design of interaction structures.

who prefer strategy $B$, then we have a network version of the Language Game of Neary (2012). Under this restriction $\gamma_{A}=\max _{i \in S^{A}} \gamma_{i}=\min _{i \in S^{A}} \gamma_{i}$ and $\gamma_{B}=$ $\max _{i \in S^{B}} \gamma_{i}=\min _{i \in S^{B}} \gamma_{i}$, so Lemma 2 and Lemma 1 [a] can be used to give necessary and sufficient conditions for stochastic stability of $\sigma^{P}$ in terms of close-knittedness of $S^{A}$ and $S^{B}$. This is captured in the following corollary.

Corollary 1. Fix a graph $\Gamma=(V, E)$ and a set of types $\left\{\gamma_{i}\right\}_{i \in V}$, such that for all $i \in V, \gamma_{i} \in\left\{\gamma_{A}, \gamma_{B}\right\}, \gamma_{B}<1 / 2<\gamma_{A}$. Profile $\sigma^{P}$ is the unique stochastically stable state if and only if $C K\left(S^{A}\right)>1-\gamma_{A}$ and $C K\left(S^{B}\right)>\gamma_{B}$.

Consider a social planner who wishes to use Lemmas 1,2 and Corollary 1 to induce a particular pattern of behavior. Suppose the planner is faced with the interaction structure and type profile in Figure 3.

As $C K\left(S^{B}\right)=3 / 8>1 / 5=\gamma_{B}=\max _{i \in S^{B}} \gamma_{i}$, we have, by [2c] of Lemma 2 , that $S^{B}$ is $\sigma_{S^{B}}^{B}$-autonomous. Consequently, all players in $S^{B}$ play $B$ at any stochastically stable state.

It remains to determine the behavior of players in $S^{A}$. We find the values of $\gamma_{A}$ such that $S^{A}$ is $\sigma_{S_{A}}^{A}$-autonomous. Note that $\gamma_{i}=\gamma_{A}$ for all $i \in S^{A}$. Consequently, $\min _{i \in S^{A}} \gamma_{i}=\max _{i \in S^{A}} \gamma_{i}=\gamma_{A}$, so [2a] and [2b] of Lemma 2 imply that $S^{A}$ is $\sigma_{S^{A}}^{A}$-autonomous if and only if $C K\left(S^{A}\right)>1-\gamma_{A}$. Computation yields that $C K\left(S^{A}\right)=3 / 10$, so we have that $S^{A}$ is $\sigma_{S^{A}}^{A}$-autonomous if and only if $\gamma_{A}>7 / 10$.

Therefore, if $\gamma_{A}>7 / 10$, then $\sigma^{P}$ is the unique stochastically stable state, as predicted by Corollary 1 . Further, it can be checked that if $\gamma_{A} \leq 7 / 10$, then not 
only is $S^{A}$ not $\sigma_{S^{A}}^{A}$-autonomous, but no subset $S \subset S^{A}$ is $\sigma_{S}^{A}$-autonomous, so by [1c] of Lemma $1, \sigma^{B}$ is stochastically stable.

Now consider the case of a social planner who wishes to induce $\sigma^{P}$ but is faced with $\gamma_{A}<7 / 10$. To overcome this problem, she would like to increase the close-knittedness of $S^{A}$ to obtain a lower threshold value of $\gamma_{A}$. Assume she has the resources to make one of three kinds of amendment: she can add an edge, delete an edge, or change the type of a player.

Adding an edge between players in $S^{B}$ will increase $C K\left(S^{B}\right)$ while leaving $C K\left(S^{A}\right)$ unaffected. Adding an edge between a player in $S^{A}$ and a player in $S^{B}$ will decrease $C K\left(S^{A}\right)$ and $C K\left(S^{B}\right)$. Adding an edge between players in $S^{A}$, for example $\left\{a_{1}, a_{3}\right\}$, will increase $C K\left(S^{A}\right)$ to $1 / 3$, which in turn lowers the threshold on $\gamma_{A}$ to $2 / 3$. If $\gamma_{A}>2 / 3$, then $\sigma^{P}$ will become uniquely stochastically stable. Conversely, if $\gamma_{A}<2 / 3$, then no single additional edge can make $\sigma^{P}$ stochastically stable.

Suppose instead that the planner deletes an edge. For this deletion to increase $C K\left(S^{A}\right)$, it must be an edge from a player in $S^{A}$ to a player in $S^{B}$, for example $\left\{a_{2}, b_{1}\right\}$. This also increases $C K\left(S^{A}\right)$ to $1 / 3$ and so lowers the threshold on $\gamma_{A}$ to $2 / 3$.

Finally, consider the planner changing the type of a single player. If she converts either $a_{1}$ or $a_{4}$ to type $\gamma_{B}$, then $C K\left(S^{A}\right)$ is reduced to $2 / 9$, whereas if she converts either $a_{2}$ or $a_{3}$, then $C K\left(S^{A}\right)$ is reduced further to $1 / 6$. Such a conversion might be useful if the planner were trying to encourage uniform adoption of strategy $B .^{3}$ In the other direction, if the planner were to convert $b_{4}$ to type $\gamma_{A}$, then $C K\left(S^{A}\right)$ would increase to $1 / 3$ and $C K\left(S^{B}\right)$ would decrease to $5 / 14$, which is small enough that $S^{B}$ would still be $\sigma_{S^{B}}^{B}$-autonomous.

\section{RANDOM PREFERENCES}

In this section we consider random preferences and give conditions under which we can usually expect any stochastically stable state to exhibit heterogeneity in strategies. Specifically, we show that as long as there is some diversity in ordinal preferences, then there will usually be diversity of behavior on sufficiently large graphs within a specific class.

First, we shall give conditions under which homogeneous states cannot

3 It should be remarked that in some contexts, the switch of a player from type $\gamma_{A}$ to type $\gamma_{B}$ could increase $C K\left(S^{A}\right)$. 
be stochastically stable when preferences are fixed. This shall depend on the plumpness of sets $S \subseteq V$, which we define as

$$
P l(S):=\frac{d(S, S)}{d(S)} .
$$

To use terminology from Young (2011), plumpness measures the area $d(S, S)$ of a set $S$ relative to its perimeter $d(S, V \backslash S){ }^{4}$

It is immediate from their definitions that $C K(S) \leq P l(S) \leq 1 / 2$. The difference in their definitions relates to potential as follows. From profile $\sigma^{B}$, if $S$ is sufficiently plump relative to $\max _{i \in S} 1-\gamma_{i}$, then potential increases if we switch $S$ to play $\sigma_{S}^{A}$. In contrast, Lemma 2 shows that, if $S$ is sufficiently close-knit, then $\sigma_{S}^{A}$ maximizes potential given $\sigma_{V \backslash S}^{B}$. In both cases, $\sigma^{B}$ is not stochastically stable.

Lemma 3. Fix a graph $\Gamma=(V, E)$ and a set of types $\left\{\gamma_{i}\right\}_{i \in V}$. Then, for any nonempty $S \subseteq V$,

[3a] If $\mathrm{Pl}(S)>\max _{i \in S} \gamma_{i}$, then $\sigma^{A}$ is not stochastically stable and there exists $S^{\prime} \subseteq S$ such that $S^{\prime}$ is $\sigma_{S^{\prime}}^{B}$-autonomous.

[3b] If $P l(S)>\max _{i \in S} 1-\gamma_{i}$, then $\sigma^{B}$ is not stochastically stable and there exists $S^{\prime} \subseteq S$ such that $S^{\prime}$ is $\sigma_{S^{\prime}}^{A}$ autonomous.

Returning to Figure 2, we see that $P l(S)=9 / 22>3 / 8=C K(S)$. Thus, for example, if $\max _{i \in S} \gamma_{i}=2 / 5$, we have that $P l(S)>\max _{i \in S} \gamma_{i}>C K(S)$. Consequently, we cannot use [2c] to state whether $S$ is $\sigma_{S}^{B}$-autonomous. That is, we do not know whether potential is always maximized when players in $S$ play $B$. However, we can use [3a] to infer that potential is higher when all players in $S$ play $B$ than when all players in $S$ play $A$. Furthermore, we know that $S$ contains a subset $S^{\prime} \subseteq S$ such that $S^{\prime}$ is $\sigma_{S^{\prime}}^{B}$-autonomous. It can be checked by calculation that the subset $S^{\prime} \subset S$ comprising the rightmost 4 vertices of $S$ is indeed $\sigma_{S^{\prime}}^{B}$-autonomous when $\max _{i \in S} \gamma_{i}=2 / 5$.

Consider a sequence of graphs $\left\{\Gamma_{k}\right\}_{k \in \mathbb{N}_{+}}, \Gamma_{k}=\left(V_{k}, E_{k}\right)$. We say that such a sequence is corpulent if, for any target level of plumpness, there is a growing

4 To see this, note that

$$
P l(S)=\frac{d(S, S)}{d(S)}=\frac{d(S, S)}{d(S, V \backslash S)+2 d(S, S)}
$$

which is increasing in the ratio of area to perimeter. 
number of non-intersecting sets of bounded size which are at least as plump as the target level.

Definition 2. A sequence of graphs $\left\{\Gamma_{k}\right\}_{k \in \mathbb{N}_{+}}$is corpulent if, for all $\phi \in(0,1 / 2)$, there exists $l \in \mathbb{N}_{+}$, such that for all $n \in \mathbb{N}_{+}$, there exists $\bar{k}$, such that for all $k \geq \bar{k}, \Gamma_{k}=\left(V_{k}, E_{k}\right)$ contains subsets $\left\{S^{m}\right\}_{m=1}^{m=n}, S^{m} \subset V_{k}$, such that $\left|S^{m}\right| \leq l$, $S^{m} \cap S^{m^{\prime}}=\emptyset$ for $m \neq m^{\prime}$, and $\mathrm{Pl}\left(S^{m}\right) \geq \phi$ for all $m$.

It follows from the definition that any corpulent sequence will be increasing in size so that $\lim _{k \rightarrow \infty}\left|V_{k}\right|=\infty$. Some examples of corpulent families of graphs are square lattices with von-Neumann neighborhood or Moore neighborhood, the Kagome lattice, and the ring (see Figure 4).

The idea of a corpulent sequence of graphs is that as the graphs in such a sequence increase in size, they include an arbitrarily large number of arbitrarily plump subsets. To illustrate this, consider the case when $\Gamma_{k}$ is the $k$ by $k$ square lattice with von-Neumann neighborhood. Assume some target level of plumpness, $\phi \in(0,1 / 2)$. Consider a subset $S$ of such a $\Gamma_{k}$, such that $S$ is composed of a $\sqrt{l}$ by $\sqrt{l}$ block of vertices so that $|S|=l$, and $d(i)=4$ for all $i \in S$ (see Figure 5). Then $d(S, S)=2 \sqrt{l}(\sqrt{l}-1)$ and $d(s)=4 l$, so $P l(S)=$ $(\sqrt{l}-1) / 2 \sqrt{l}$. This implies that if we choose $l$ large enough, then $P l(S)>\phi$. For any positive integer $n$, we can then choose $k$ large enough that $\Gamma_{k}$ includes $n$ such sets $S^{1}, S^{2}, \ldots, S^{n}$ that do not intersect one another, thus satisfying the definition of corpulence.

Now, let each $\gamma_{i}, i \in V$, be independently drawn according to a probability measure $\mathbb{P}$ on the Borel sets of $(0,1)$. We say that preferences are ordinally diverse if there is nonzero probability of a given player $i \in V$ having an ordinal preference for $A$ over $B$ and vice versa.

Definition 3. Preferences are ordinally diverse if $\mathbb{P}\left[\gamma_{i} \in(0,1 / 2)\right]>0$ and $\mathbb{P}\left[\gamma_{i} \in\right.$ $(1 / 2,1)]>0$.

For a given graph $\Gamma=(V, E)$, abuse notation to let $\mathbb{P}[\mathscr{H} \mid \Gamma]$ be the probability that one of the two homogeneous states, $\sigma^{A}$ or $\sigma^{B}$, is stochastically stable when the types $\left\{\gamma_{i}\right\}_{i \in V}$ are determined according to $\mathbb{P}$.

We can now state the main result of this section. When preferences are ordinally diverse, large corpulent graphs will nearly always have heterogeneity in strategies at stochastically stable states. That is, ordinal diversity in preferences implies diversity in behavior for large graphs within these families. 


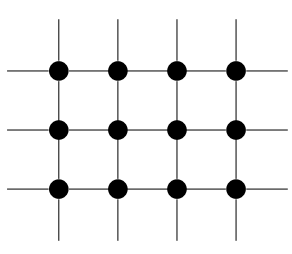

(a)

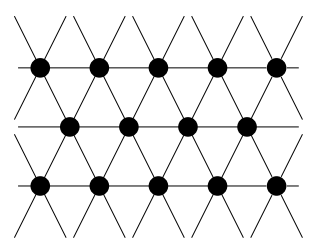

(c)

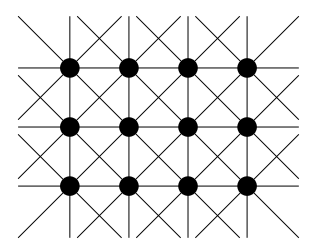

(b)

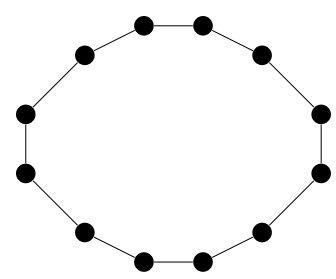

(d)

Figure 4: Examples of corpulent families of graphs include (a) square lattice with von-Neumann neighborhood; (b) square lattice with Moore neighborhood; (c) Kagome lattice; (d) the ring.

Theorem 1. If $\left\{\Gamma_{k}\right\}_{k \in \mathbb{N}_{+}}$is corpulent and preferences are ordinally diverse then $\lim _{k \rightarrow \infty} \mathbb{P}\left[\mathscr{H} \mid \Gamma_{k}\right]=0$.

The intuition behind the theorem is that large graphs in corpulent sequences have large numbers of very plump sets of vertices. Indeed, for an arbitrary target level of plumpness it is possible to choose a graph large enough that it has an arbitrary number of non-intersecting sets that are at least as plump as the target level. Ordinal diversity ensures that, usually, at least some of these sets will be composed solely of players with an ordinal preference in favour of strategy $A$ and some will be composed solely of players with an ordinal preference for strategy $B$. These preferences may be cardinally very weak, but this does not matter as the target level of plumpness can be adjusted to take account of this. Consequently, large corpulent graphs under random preferences will usually contain sets of players with homogeneous ordinal preferences that are plump enough, per Lemma 3, to destabilize homogeneous behavior in the population. 




Figure 5: Non-intersecting sets $S^{1}, S^{2}$ such that $l=\left|S^{1}\right|=\left|S^{2}\right|=16$ and $P l\left(S^{1}\right)=P l\left(S^{2}\right)=3 / 8$. Arbitrarily large square lattices with von-Neumann neighborhood can include arbitrarily large numbers of such sets.

\section{A. APPENDIX}

Proof of Lemma 1 [a].

$(\Leftarrow)$ Assume that $S^{A}$ is $\sigma_{S^{A}}^{A}$-autonomous and $S^{B}$ is $\sigma_{S^{B}}^{B}$-autonomous. As $S^{A}$ is $\sigma_{S^{A}}^{A}$-autonomous, any state $\sigma^{*}$ that maximizes potential and is thus stochastically stable must, by Definition 1 , have $\sigma_{S^{A}}^{*}=\sigma_{S^{A}}^{A}$. Similarly, $\sigma_{S^{B}}^{*}=\sigma_{S^{B}}^{B}$. Therefore, $\sigma^{*}=\left(\sigma_{S^{A}}^{A}, \sigma_{S^{B}}^{B}\right)=\sigma^{P}$.

$(\Rightarrow)$ Assume that $\sigma^{P}$ is uniquely stochastically stable and thus uniquely maximizes potential. If $S^{A}$ is not $\sigma_{S^{A}}^{A}$-autonomous, then, by Definition 1 , for some $\sigma, \sigma_{S^{A}} \neq \sigma_{S^{A}}^{A}$,

$$
\operatorname{Potential}\left(\sigma_{S^{A}}^{A}, \sigma_{-S^{A}}\right) \leq \operatorname{Potential}(\sigma) .
$$

Note that, by (2), edges between vertices playing different strategies give lower potential than edges between vertices playing the same strategy. Therefore, (3) implies

$$
\operatorname{Potential}\left(\sigma_{S^{A}}^{A}, \sigma_{-S^{A}}^{B}\right) \leq \operatorname{Potential}\left(\sigma_{S^{A}}, \sigma_{-S^{A}}^{B}\right) .
$$

but as $\sigma^{P}=\left(\sigma_{S^{A}}^{A}, \sigma_{-S^{A}}^{B}\right)$, inequality (4) implies that $\sigma^{P}$ does not uniquely maximize potential, so $\sigma^{P}$ is not uniquely stochastically stable. Contradiction. Therefore, $S^{A}$ must be $\sigma_{S^{A}}^{A}$-autonomous. Similarly, $S^{B}$ must be $\sigma_{S^{B}}^{B}$-autonomous. 
Proof of Lemma 1[b] (and by analogy, [c]).

$(\Rightarrow)$ Assume that $\sigma^{A}$ is stochastically stable and thus maximizes potential. If there exists $S \subseteq V$ such that $S$ is $\sigma_{S}^{B}$-autonomous, then by Definition 1,

$$
\operatorname{Potential}\left(\sigma_{S}^{B}, \sigma_{-S}^{A}\right)>\operatorname{Potential}\left(\sigma^{A}\right),
$$

contradicting $\sigma^{A}$ being a potential maximizer. Therefore, there does not exist $S \subseteq V$ such that $S$ is $\sigma_{S}^{B}$-autonomous.

$(\Leftarrow)$ Assume that there does not exist $S \subseteq V$ such that $S$ is $\sigma_{S}^{B}$-autonomous. If $\sigma^{A}$ is not stochastically stable then it does not maximize potential. Amongst all maximizers of potential, choose one, denoted $\sigma^{*}$, such that, denoting $S=\left\{i \in V: \sigma_{i}^{*}=B\right\}$, for any $S^{\prime} \subset S, \sigma^{\prime}=\left(\sigma_{S^{\prime}}^{B}, \sigma_{-S^{\prime}}^{A}\right)$ does not maximize potential. Then we have that for all $\sigma_{S} \neq \sigma_{S}^{B}$,

$$
\operatorname{Potential}\left(\sigma^{*}\right)=\operatorname{Potential}\left(\sigma_{S}^{B}, \sigma_{-S}^{A}\right)>\operatorname{Potential}\left(\sigma_{S}, \sigma_{-S}^{A}\right)
$$

Note that by (2), edges between vertices playing different strategies give lower potential than edges between vertices playing the same strategy. Therefore, (6) implies that for any $\sigma, \sigma_{S} \neq \sigma_{S}^{B}$,

$$
\operatorname{Potential}\left(\sigma_{S}^{B}, \sigma\right)>\operatorname{Potential}(\sigma)
$$

which is the definition of $S$ being $\sigma^{B}$-autonomous. Contradiction. Therefore, $\sigma^{A}$ is stochastically stable.

Proof of Lemma 2[a] (and by analogy, [c]).

Assume that $C K(S)>\max _{i \in S} 1-\gamma_{i}$. Note that as, by (2), edges between vertices playing different strategies give lower potential than edges between vertices playing the same strategy, $S$ is $\sigma_{S}^{A}$-autonomous if and only if for all $S^{\prime} \subseteq S$,

$$
\operatorname{Potential}\left(\sigma_{S}^{A}, \sigma_{V \backslash S}^{B}\right)>\operatorname{Potential}\left(\sigma_{S \backslash S^{\prime}}^{A}, \sigma_{S^{\prime}}^{B}, \sigma_{V \backslash S}^{B}\right) .
$$


Substituting from (2),

$$
\begin{aligned}
& \text { Potential }\left(\sigma_{S}^{A}, \sigma_{V \backslash S}^{B}\right)-\text { Potential }\left(\sigma_{S \backslash S^{\prime}}^{A}, \sigma_{S^{\prime}}^{B}, \sigma_{V \backslash S}^{B}\right) \\
& =\sum_{i \in S^{\prime}} d(i, V \backslash S)\left(\gamma_{i}-1\right)+\sum_{i \in S^{\prime}} d\left(i, S \backslash S^{\prime}\right)\left(\gamma_{i}\right)+\sum_{i, j \in S^{\prime}}\left(\gamma_{i}+\gamma_{j}-1\right) . \\
& >d\left(S^{\prime}, V \backslash S\right)\left(\min _{i \in S^{\prime}} \gamma_{i}-1\right)+d\left(S^{\prime}, S \backslash S^{\prime}\right)\left(\min _{i \in S^{\prime}} \gamma_{i}\right) \\
& \quad+d\left(S^{\prime}, S^{\prime}\right)\left(2 \min _{i \in S^{\prime}} \gamma_{i}-1\right) .
\end{aligned}
$$

Now,

$$
C K(S)>\max _{i \in S} 1-\gamma_{i}=1-\min _{i \in S} \gamma_{i}
$$

so by definition of $C K(S)$, we have, for all $S^{\prime} \subseteq S$,

$$
\begin{aligned}
& \frac{d\left(S^{\prime}, S\right)}{d\left(S^{\prime}\right)}>1-\min _{i \in S} \gamma_{i} \\
& \Longrightarrow d\left(S^{\prime}, S\right)-d\left(S^{\prime}\right)\left(1-\min _{i \in S} \gamma_{i}\right)>0 \\
& \Longrightarrow d\left(S^{\prime}, S\right)-d\left(S^{\prime}\right)\left(1-\min _{i \in S^{\prime}} \gamma_{i}\right)>0 \\
& \Longrightarrow \underbrace{d\left(S^{\prime}, S \backslash S^{\prime}\right)+d\left(S^{\prime}, S^{\prime}\right)}_{=d\left(S^{\prime}, S\right)} \\
& \quad-\underbrace{\left(d\left(S^{\prime}, V \backslash S\right)+d\left(S^{\prime}, S \backslash S^{\prime}\right)+2 d\left(S^{\prime}, S^{\prime}\right)\right)}_{=d\left(S^{\prime}\right)}\left(1-\min _{i \in S^{\prime}} \gamma_{i}\right)>0 \\
& \Longrightarrow d\left(S^{\prime}, V \backslash S\right)\left(\min _{i \in S^{\prime}} \gamma_{i}-1\right)+d\left(S^{\prime}, S \backslash S^{\prime}\right)\left(\min _{i \in S^{\prime}} \gamma_{i}\right) \\
& \quad+d\left(S^{\prime}, S^{\prime}\right)\left(2 \min _{i \in S^{\prime}} \gamma_{i}-2\right)>0 .
\end{aligned}
$$

So (9) and (11) together imply that for all $S^{\prime} \subseteq S$,

$$
\text { Potential }\left(\sigma_{S}^{A}, \sigma_{V \backslash S}^{B}\right)-\operatorname{Potential}\left(\sigma_{S \backslash S^{\prime}}^{A}, \sigma_{S^{\prime}}^{B}, \sigma_{V \backslash S}^{B}\right)>0,
$$

which implies (8), so $S$ is $\sigma_{S}^{A}$-autonomous. 
Proof of Lemma 2[b] (and by analogy, [d]).

Assume that $S$ is $\sigma_{S}^{A}$-autonomous. Then for all $S^{\prime} \subseteq S$, (8) holds, so

$$
\begin{aligned}
& \text { Potential }\left(\sigma_{S}^{A}, \sigma_{V \backslash S}^{B}\right)-\operatorname{Potential}\left(\sigma_{S \backslash S^{\prime}}^{A}, \sigma_{S^{\prime}}^{B}, \sigma_{V \backslash S}^{B}\right)>0 \\
& \Longrightarrow \sum_{i \in S^{\prime}} d(i, V \backslash S)\left(\gamma_{i}-1\right)+\sum_{i \in S^{\prime}} d\left(i, S \backslash S^{\prime}\right)\left(\gamma_{i}\right)+\sum_{i, j \in S^{\prime}}\left(\gamma_{i}+\gamma_{j}-1\right)>0 \\
& \Longrightarrow d\left(S^{\prime}, V \backslash S\right)\left(\max _{i \in S^{\prime}} \gamma_{i}-1\right)+d\left(S^{\prime}, S \backslash S^{\prime}\right)\left(\max _{i \in S^{\prime}} \gamma_{i}\right) \\
& \quad+d\left(S^{\prime}, S^{\prime}\right)\left(2 \max _{i \in S^{\prime}} \gamma_{i}-1\right)>0 \\
& \Longrightarrow d\left(S^{\prime}, S\right)-d\left(S^{\prime}\right)\left(1-\max _{i \in S^{\prime}} \gamma_{i}\right)>0 \quad[\text { by similar algebra to (11)] } \\
& \Longrightarrow d\left(S^{\prime}, S\right)-d\left(S^{\prime}\right)\left(1-\max _{i \in S} \gamma_{i}\right)>0 \\
& \Longrightarrow \frac{d\left(S^{\prime}, S\right)}{d\left(S^{\prime}\right)}>1-\max _{i \in S} \gamma_{i},
\end{aligned}
$$

which implies that

$$
C K(S)>1-\max _{i \in S} \gamma_{i}=\min _{i \in S} 1-\gamma_{i}
$$

Proof of Corollary 1.

Note that

$$
\min _{i \in S^{A}} \gamma_{i}=\gamma_{A}=\max _{i \in S^{A}} \gamma_{i}, \quad \min _{i \in S^{B}} \gamma_{i}=\gamma_{B}=\max _{i \in S^{B}} \gamma_{i}
$$

Therefore, by Lemma 2[a,b], $S^{A}$ is $\sigma_{S^{A}}^{A}$-autonomous if and only if $C K\left(S^{A}\right)>$ $1-\gamma_{A}$.

Similarly, by Lemma 2[c,d], $S^{B}$ is $\sigma_{S^{B}}^{B}$-autonomous if and only if $C K\left(S^{B}\right)>\gamma_{B}$. So $S^{A}$ is $\sigma_{S^{A}}^{A}$-autonomous and $S^{B}$ is $\sigma_{S^{B}}^{B}$-autonomous if and only if $C K\left(S^{A}\right)>$ $1-\gamma_{A}$ and $C K\left(S^{B}\right)>\gamma_{B}$.

By Lemma 1[a], $S^{A}$ is $\sigma_{S^{A}}^{A}$-autonomous and $S^{B}$ is $\sigma_{S^{B}}^{B}$-autonomous if and only if $\sigma^{P}$ is the unique stochastically stable state. 
Proof of Lemma 3[a] (and by analogy, [b]).

We have

$$
\begin{aligned}
& P l(S)=\frac{d(S, S)}{d(S)}>\max _{i \in S} \gamma_{i} \\
& \Longrightarrow d(S) \max _{i \in S} \gamma_{i}-d(S, S)<0 .
\end{aligned}
$$

Now the potential difference between $\sigma^{A}$ and $\left(\sigma_{S}^{B}, \sigma_{V \backslash S}^{A}\right)$ equals

$$
\begin{aligned}
& \operatorname{Potential}\left(\sigma^{A}\right)-\operatorname{Potential}\left(\sigma_{S}^{B}, \sigma_{V \backslash S}^{A}\right) \\
& =\sum_{i \in S} d(i, V \backslash S) \gamma_{i}+\sum_{i, j \in S} \gamma_{i}+\gamma_{j}-1 \\
& \leq d(S, V \backslash S) \max _{i \in S} \gamma_{i}+d(S, S)\left(2 \max _{i \in S} \gamma_{i}-1\right) \\
& =\underbrace{(d(S)-2 d(S, S))}_{=d(S, V \backslash S)} \max _{i \in S} \gamma_{i}+d(S, S)\left(2 \max _{i \in S} \gamma_{i}-1\right) \\
& =d(S) \max _{i \in S} \gamma_{i}-d(S, S) \\
& \underbrace{<}_{\text {by }(16)} 0 .
\end{aligned}
$$

Therefore, $\sigma^{A}$ does not maximize potential and is thus not stochastically stable. Consider $\sigma_{S}$ that maximize potential given $\sigma_{V \backslash S}=\sigma_{V \backslash S}^{A}$. Consider such a $\sigma_{S}$, denoted $\sigma_{S}^{*}$, such that, denoting $S^{*}=\left\{i \in S: \sigma_{i}^{*}=B\right\} \subseteq S$, for any $S^{\prime} \subset S^{*}$, $\sigma^{\prime}=\left(\sigma_{S^{\prime}}^{B}, \sigma_{-S^{\prime}}^{A}\right)$ does not maximize potential. Then we have that, for all $\sigma_{S^{*}} \neq \sigma_{S^{*}}^{B}$

$$
\operatorname{Potential}\left(\sigma_{S^{*}}^{B}, \sigma_{-S^{*}}^{A}\right)>\operatorname{Potential}\left(\sigma_{S}, \sigma_{-S^{*}}^{A}\right) .
$$

Note that by (2), edges between vertices playing different strategies give lower potential than edges between vertices playing the same strategy. Therefore, (6) implies that for any $\sigma, \sigma_{S^{*}} \neq \sigma_{S^{*}}^{B}$,

$$
\operatorname{Potential}\left(\sigma_{S^{*}}^{B}, \sigma\right)>\operatorname{Potential}(\sigma)
$$

which is the definition of $S^{*}$ being $\sigma^{B}$-autonomous. 
Proof of Theorem 1.

As preferences are assumed to be ordinally diverse, by Definition 3, there exists $\phi<1 / 2$ such that $\mathbb{P}[(0, \phi)]=: \rho>0$.

As $\left\{\Gamma_{k}\right\}_{k \in \mathbb{N}_{+}}$is corpulent, by Definition 2 there exists $l$ such that for all $n \in \mathbb{N}_{+}$, there exists $\bar{k}(n)$ such that for $k \geq \bar{k}(n)$ we can choose non-intersecting sets $\left\{S^{m}\right\}_{1 \leq m \leq n},\left|S^{m}\right| \leq l$ such that $\operatorname{Pl}\left(S^{m}\right) \geq \phi$.

For given $\Gamma_{k}, S^{m}$, as $\left|S^{m}\right| \leq l$, the probability that all $i \in S^{m}$ have $\gamma_{i}<\phi$, and hence $\phi>\max _{i \in S^{m}}$, is bounded below by $\rho^{l}>0$. The probability that this holds for at least one such $S^{m}$ is thus bounded below by $1-\left(1-\rho^{l}\right)^{n}$ for $k \geq \bar{k}$. This probability approaches one as $n \rightarrow \infty$.

So, with probability approaching one as $k \rightarrow \infty$, there exists $S^{m} \subseteq V_{k}$ such that $P l\left(S^{m}\right) \geq \phi>\max _{i \in S^{m}} \gamma_{i}$, and by Lemma $3, \sigma^{A}$ is not stochastically stable. A similar argument holds for $\sigma^{B}$.

\section{References}

Blume, L. E. (1993). The statistical mechanics of strategic interaction. Games and Economic Behavior, 5(3), 387 - 424.

Blume, L. E. (1996). Population games (Working Papers No. 96-04-022). Santa Fe Institute.

Ellison, G. (2000). Basins of attraction, long-run stochastic stability, and the speed of step-by-step evolution. Review of Economic Studies, 67(1), 17-45.

Foster, D., \& Young, H. P. (1991). Cooperation in the long-run. Games and Economic Behavior, 3(1), 145-156.

Freidlin, M. I., \& Wentzell, A. D. (1984). Random Perturbations of Dynamical Systems. Springer.

Granovetter, M. (1978). Threshold models of collective behavior. American Journal of Sociology, 83(6), pp. 1420-1443.

Kandori, M., Mailath, G. J., \& Rob, R. (1993). Learning, mutation, and long run equilibria in games. Econometrica, 61(1), 29-56.

Lewis, D. (1969). Convention: A Philosophical Study. Harvard University Press.

Monderer, D., \& Shapley, L. S. (1996). Potential games. Games and Economic Behavior, 14(1), 124 - 143.

Montanari, A., \& Saberi, A. (2010). The spread of innovations in social networks. Proceedings of the National Academy of Sciences, 107(47), 20196-20201.

Neary, P. R. (2012). Competing conventions. Games and Economic Behavior, 76(1), 301-328. 
Newton, J., \& Angus, S. D. (2015). Coalitions, tipping points and the speed of evolution. Journal of Economic Theory, 157, 172 - 187.

Newton, J., \& Sercombe, D. (2017). Agency, potential and contagion. mimeo.

Peski, M. (2010). Generalized risk-dominance and asymmetric dynamics. Journal of Economic Theory, 145(1), 216 - 248.

Staudigl, M. (2012). Stochastic stability in asymmetric binary choice coordination games. Games and Economic Behavior, 75(1), 372 - 401.

Young, H. P. (1993). The evolution of conventions. Econometrica, 61(1), 57-84.

Young, H. P. (2011). The dynamics of social innovation. Proceedings of the National Academy of Sciences, 108 Suppl 4, 21285-91. 